\title{
Single- versus two-port video-assisted thoracic surgery in mediastinal tumor: a propensity-matched study
}

\author{
Shilong Wu ${ }^{1 \#}$, Hengrui Liang ${ }^{1 \#}$, Wenhua Liang ${ }^{1 \#}$, Yaoliang Zhang', Yanzhi Ma ${ }^{3}$, Hui Liu ${ }^{2}$, Hanyu Yang ${ }^{2}$, \\ Jun Liu ${ }^{1}$, Jianxing $\mathrm{He}^{1}$ \\ ${ }^{1}$ Department of Thoracic Surgery and Oncology, the First Affiliated Hospital of Guangzhou Medical University, State Key Laboratory of \\ Respiratory Disease, National Clinical Research Center for Respiratory Disease, Guangzhou Institute of Respiratory Health, Guangzhou 510120, \\ China; ${ }^{2}$ Department of Anesthesia, the First Affiliated Hospital of Guangzhou Medical University, Guangzhou 510120, China; ${ }^{3}$ Guangzhou Medical \\ University, Guangzhou 510120, China \\ Contributions: (I) Conception and design: S Wu, H Liang; (II) Administrative support: J He, Jun Liu; (III) Provision of study materials or patients: H \\ Liang, W Liang; (IV) Collection and assembly of data: S Wu, H Liang, Y Zhang; (V) Data analysis and interpretation: S Wu, Y Ma; (VI) Manuscript \\ writing: All authors; (VII) Final approval of manuscript: All authors. \\ \#These authors contributed equally to this work. \\ Correspondence to: Jun Liu, MD, PhD; Jianxing He, MD, PhD, FACS. Department of Thoracic Surgery and Oncology, the First Affiliated Hospital \\ of Guangzhou Medical University, State Key Laboratory of Respiratory Disease, National Clinical Research Center for Respiratory Disease, \\ Guangzhou Institute of Respiratory Health, No. 151, Yanjiang Rd., Guangzhou 510120, China. Email: liujun9707@sina.com; drjianxing.he@gmail.com.
}

Background: In this retrospective study, we aimed to compare single-port (SP) and two-port (TP) videoassisted thoracic surgery (VATS) for the surgical resection of mediastinal tumor.

Methods: Between December 2015 and October 2018, a total of 191 consecutive mediastinal tumor patients who underwent SP- or TP-VATS in the First Affiliated Hospital of Guangzhou Medical University were enrolled. Propensity-matched analysis, incorporating preoperative clinical features, was used to compare the perioperative outcomes and analyse the safety and efficacy between SP-VATS and TP-VATS for mediastinal tumor.

Results: There were 43 patients in the SP-VATS group, and 148 patients in the TP-VATS group from December 2015 to October 2018. Propensity matching produced 40 pairs in this retrospective study. The baseline characters were all well balanced. During the operation, the SP-VATS group showed shorter operation time (89.38 vs. $101.82 \mathrm{~min} ; \mathrm{P}=0.042)$ and less chest tube [16 (40.0\%) vs. 28 (70.0\%); $\mathrm{P}=0.007]$ than TP-VATS group. Postoperatively, SP and TP groups showed similar results in terms of morbidity and recovery.

Conclusions: In this retrospective study, our experiences have shown that SP-VATS is a safe and promising technique and is not inferior to the TP-VATS.

Keywords: Single-port; two-port; video-assisted thoracic surgery (VATS); mediastinal tumor

Submitted Aug 01, 2019. Accepted for publication Oct 31, 2019.

doi: $10.21037 /$ jtd.2019.11.05

View this article at: http://dx.doi.org/10.21037/jtd.2019.11.05

\section{Introduction}

The median sternotomy is the traditional surgical approach for mediastinal tumor resection. Patients will recover slowly and have many complications, for the median sternotomy can lead to large trauma $(1,2)$. Compared to thoracotomy, video-assisted thoracic surgery (VATS) has been associated with shorter length of hospital stay and fewer complications including major cardiopulmonary complications, hemorrhage, respiration and atrial arrhythmia (3), less pain and a better quality of life (4).

In 1992, VATS burst into the thoracic surgery $(5,6)$. Yim et al. showed their experiences about three-port 


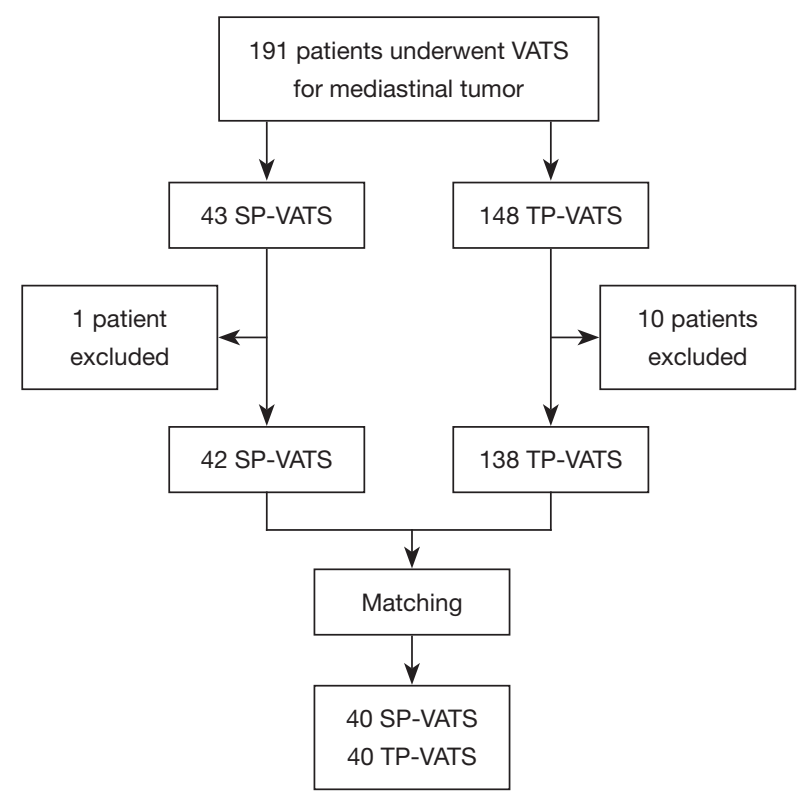

Figure 1 Schema of patient grouping and matching. SP, singleport; TP, two-port; VATS, video-assisted thoracic surgery.

VATS in 1994 (7). In 1998, Yamamoto et al. used a 2-cm skin incision to perform lung resection successfully (8). The VATS procedure has made evolution gradually from classical three-port VATS to eventually single-port VATS (SP-VATS). Reduced surgical traumas with less pain, shorter hospital stay, decreased morbidity, better cosmesis and faster recovery are the most relevant features by the numerous VATS operations (9).

Now, SP-VATS is the "talk of the town" in minimally invasive surgery and widely used in various thoracic surgeries. Some studies have compared SP-VATS with two-port VATS (TP-VATS) in various thoracic surgeries including lobectomy and pneumothorax $(10,11)$. However, there were very few reports about SP-VATS versus TPVATS in mediastinal tumor resection $(12,13)$. What's more, few researches have been compared immune-inflammation response in different ports of VATS. Further study is worth being done to compare the two techniques in mediastinal tumor resection. Our study aimed to compare the shortterm outcomes and immune-inflammation response of SPVATS versus TP-VATS in mediastinal surgeries.

\section{Methods}

\section{Patients}

The patients underwent SP- or TP-VATS for mediastinal tumor from December 2015 to October 2018 in the First Affiliated Hospital of Guangzhou Medical University were retrospectively reviewed. The inclusion criteria were found mediastinal tumor on preoperative imaging and underwent SP- or TP-VATS. The exclusion criteria were as follows: received bilateral surgery to both sides, conversion to thoracotomy or median sternotomy, invaded to the surrounding organs obviously, with a history of chest surgery, with an American Society of Anesthesiologists (ASA) score of IV-VI. There was one patient conversion to median sternotomy in each group because of massive hemorrhage. Seven patients' lesions clearly invaded surrounding organs and four patients had serious adhesions in TP-VATS group (Figure 1). The selection of surgical procedures was based on surgeon's discretion and patients' wishes. All patients had signed the consent before the surgery.

\section{Surgical procedures}

The operative procedures of SP-VATS were as follows: if the patients had anterior mediastinum tumor, the patients were positioned in a $30^{\circ}$ semisupine position with a roll under the shoulder and the ipsilateral arm held abducted over a padded L-screen to expose the axilla for port placement. If the patients had posterior mediastinum tumor, the ipsilateral side of the chest was elevated by approximately $30^{\circ}$. The contralateral arm placed under the neck and the ipsilateral arm was hung on an overhead padded holder to expose the axilla. If the tumour is located at the middle mediastinum, the patients were positioned in the lateral decubitus position. A 2- to 4-cm wound was created in the 3 rd to 7 th intercostal space at the anterior or mid-axillary line. A 30 angled camera was placed in the lower lateral. All procedures were performed under thoracoscope. The specimen was retrieved by a specimen bag through the incision wound.

The differences between SP-VATS and TP-VATS were that: we created 2 wounds, one for a $30^{\circ}$ angled camera and another working port for the endoscopic instruments. The position of the utility and camera port was determined the location of tumor. Incision length was determined the size of the lesion. At the end of the surgery, a 24-F chest tube was placed at the end of incision wound.

\section{Postoperative management}

Patients were admitted to the ward or intensive care unit 
(ICU) after regained consciousness in Post Anesthesia Care Unit. Checking routine blood, D-dimer and arterial blood gas analysis after backing from the ward or ICU. Analgesics are used according to the doctor's comprehensive assessment and the patients' feelings. X-ray chest plain film or B-mode ultrasonographic scanning was performed after operation. The chest tube was removed when no air leak was observed and total drainage less than $100 \mathrm{~mL}$ in 24 hours. Patients discharged criteria were as follows: normal vital signs, no complications requiring in-hospital treatment, lung re-expansion $>70 \%$ after the chest tube removal.

\section{Date collection and statistical analysis}

Patients' data include age, gender, body mass index (BMI), forced expiratory volume in one second (FEV1), preoperative coagulation function, preoperative routine blood examination results [absolute counts of leukocytes, macrophages, neutrophils, lymphocytes and platelets (PLTs)], incision laterality, tumor location, tumor size, pathological type and ASA status. The patients were divided into two groups according to the incision number. A propensity score was performed for 180 patients between SP-VATS and TP-VATS with SPSS 24.0. Patients were matched at 1:1 in the two groups by a nearest-neighbour method. The propensity score was calculated by using age, gender, BMI, FEV1, incision laterality, tumor location and tumor size.

Perioperative features, including postoperative coagulation function and arterial blood gas index, immuneinflammation index (absolute counts of leukocytes, macrophages, neutrophils, lymphocytes and PLTs), postoperative complication, operation time, intraoperative blood loss, perioperative mortality, chest tube, chest tube duration and volume, hospital stays after surgery and postoperative analgesia medication were recorded. The postoperative complications were evaluated by the Common Terminology Criteria for Adverse Events version 3.0 (14). The SII (the systemic immune-inflammation index) was calculated by using the following formula: SII = PLT count $\times$ neutrophil count/lymphocyte count.

Data were presented as mean value \pm standard deviation or median [interquartile range (IQR)] for continuous variables, and percentages for categorical variables. Continuous variables were analyzed using Student's $t$-test or Mann-Whitney U-test, and categorical variables were analyzed using the Pearson's $\chi^{2}$ test or Fisher's exact test.
All $\mathrm{P}$ values were bilaterally distributed, and $\mathrm{P}<0.05$ was considered statistically significant. All of the analyses were performed on the SPSS statistical software package 24.0 (IBM Corp, Armonk, NY, USA).

\section{Statement of ethics approval}

The National Key R\&D Program of China evaluated the protocol. The data involved in this study was collected retrospectively, which was not required the statement of ethics approval.

\section{Results}

\section{Patient characteristics}

A total of 180 patients between December 2015 and November 2018 were consecutively included in this analysis, $42(23.33 \%)$ patients underwent SP-VATS and 138 (76.67\%) patients underwent TP-VATS. The mean age was $47.09 \pm 14.66$ years, and the mean tumor size was $6.02 \pm 3.17 \mathrm{~cm}$. After 1:1 propensity score matching (PSM), baseline demographic and clinical variables were well balanced between the two groups (Table 1).

\section{Operative results and postoperative recovery}

No death occurred during the perioperative period. None of patients conversed from SP-VATS to TP-VATS and none of patients conversed from TP-VATS to more port VATS. There was one patient conversion to median sternotomy in each group. Compared with TP-VATS group, patients in SP-VATS experienced shorter operation time [mean, IQR: 89.38, 51.25-98.75 vs. 101.82 , 65.00-123.75 min; $\mathrm{P}=0.042]$ and less chest tube [16 (40.0\%) vs. 28 (70.0\%); $\mathrm{P}=0.007$ ]. Less chest tube means fewer patients placed chest tube. The SP-VATS group had less intraoperative blood loss (mean, IQR: $31.03,10.00-20.00$ vs. 85.35, 10.00-50.00 mL; $\mathrm{P}=0.109)$ and more total drainage volume $(434.13 \pm 394.30$ vs. $318.82 \pm 226.32 \mathrm{~mL} ; \mathrm{P}=0.296$ ), but without significant difference. The drainage duration $(0.90 \pm 1.36$ vs. $1.45 \pm 1.28$ days; $\mathrm{P}=0.066$ ), hospital stays after surgery (mean, IQR: 3, 2-5.75 vs. 3, 2-5 days; $\mathrm{P}=0.953$ ), post-operative arterial blood gas were similar between the two groups. Postoperative complications occurred in 2 patients $(5.0 \%)$ in the SP-VATS group and 4 patients $(10.0 \%)$ of patients in the TP-VATS group, without any significant differences $(\mathrm{P}=0.392)$. There were two cases of pleural fluid leakage in 
Table 1 Clinical features before and after matching

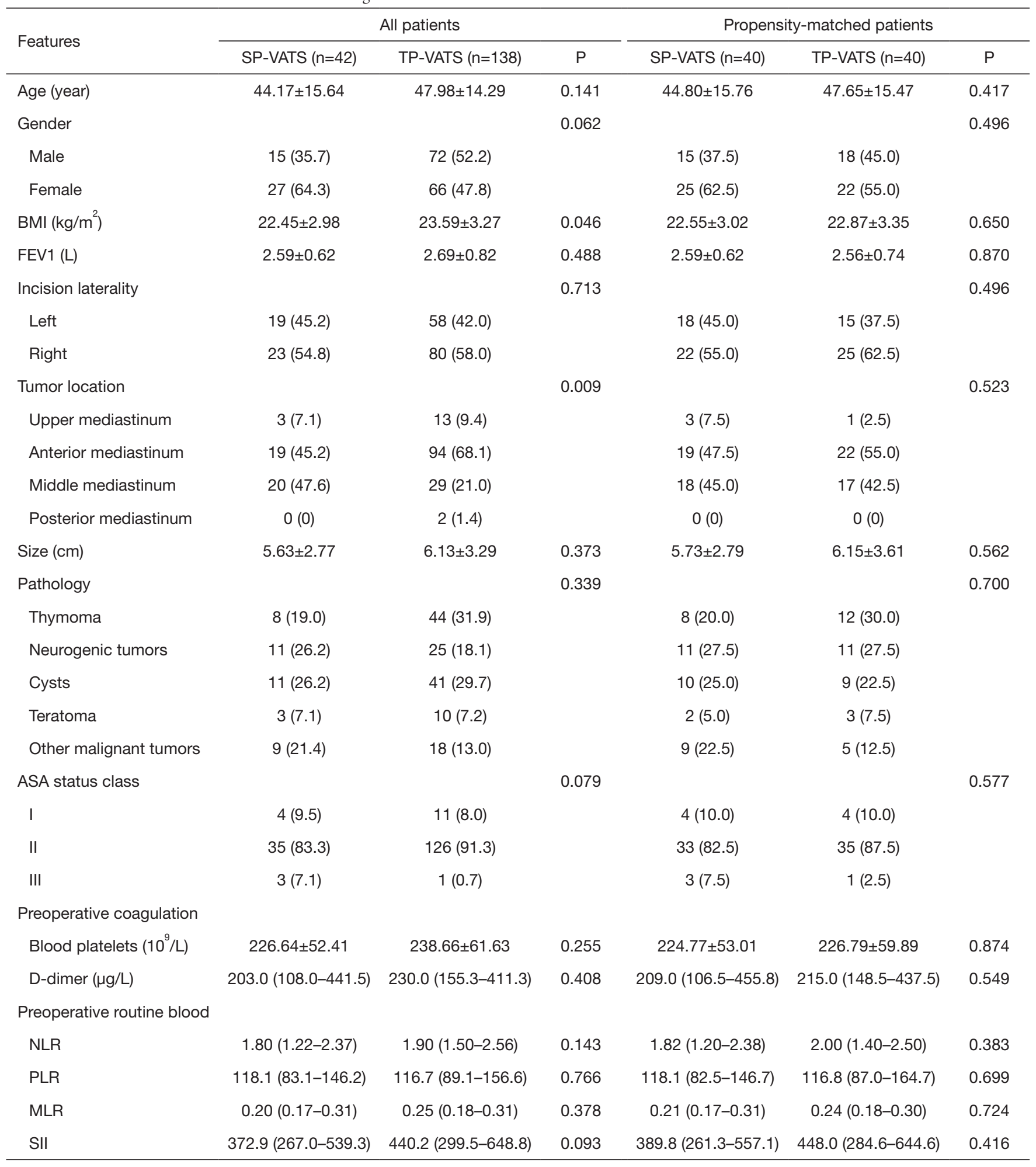

Discrete data are expressed as number with percentages: $n$ (\%); continuous data are expressed as mean \pm SD or median (interquartile range). SP, single-port; TP, two-port; VATS, video-assisted thoracic surgery; BMI, body mass index; FEV1, forced expiratory volume in the first second; ASA, American Society of Anesthesiologists; NLR, neutrophil/lymphocyte ratio; PLR, platelet/lymphocyte ratio; MLR, macrophages/lymphocyte ratio; SII, the systemic immune-inflammation index. 
Table 2 Comparison on perioperative outcomes

\begin{tabular}{|c|c|c|c|}
\hline Variables & SP-VATS $(n=40)$ & TP-VATS $(n=40)$ & $P$ \\
\hline $\operatorname{PLT}\left(10^{9} / \mathrm{L}\right)$ & $204.18 \pm 36.91$ & $199.20 \pm 48.47$ & 0.621 \\
\hline D-dimer ( $\mu \mathrm{g} / \mathrm{L})$ & $1,425[419-1,992]$ & $956[379-1,141]$ & 0.387 \\
\hline \multicolumn{4}{|l|}{ Post-operative arterial blood gas } \\
\hline $\mathrm{PaCO}_{2}(\mathrm{mmHg})$ & $40.04 \pm 6.35$ & $44.67 \pm 7.37$ & 0.199 \\
\hline $\mathrm{HCO}_{3}{ }^{-}(\mathrm{mmol} / \mathrm{L})$ & $23.87 \pm 5.56$ & $22.49 \pm 4.84$ & 0.237 \\
\hline Complication & $2(5.0)$ & $4(10.0)$ & 0.392 \\
\hline Operation time (min) & 89.38 (51.25-98.75) & $101.82(65.00-123.75)$ & 0.042 \\
\hline Drainage duration (days) & $0.90 \pm 1.36$ & $1.45 \pm 1.28$ & 0.066 \\
\hline Total drainage volume (mL) & $434.13 \pm 394.30$ & $318.82 \pm 226.32$ & 0.296 \\
\hline Hospital stays after surgery (day) & $3(2-5.75)$ & 3 [2-5] & 0.953 \\
\hline \multicolumn{4}{|l|}{ Inflammation marker } \\
\hline NLR & $8.67(5.15-18.55)$ & $11.42(6.64-17.13)$ & 0.262 \\
\hline PLR & $177.4(129.7-243.4)$ & $232.0(160.9-303.8)$ & 0.073 \\
\hline MLR & $0.50(0.32-0.74)$ & $0.57(0.36-0.86)$ & 0.328 \\
\hline SII & $1,629.4(1,011.9-3,698.6)$ & 2,053.3 (1,610.0-3,800.0) & 0.359 \\
\hline
\end{tabular}

Discrete data are expressed as number with percentages: $n$ (\%); continuous data are expressed as mean \pm SD or median (interquartile range). SP, single-port; TP, two-port; VATS, video-assisted thoracic surgery; PLT, platelet; NLR, neutrophil/lymphocyte ratio; PLR, platelet/ lymphocyte ratio; MLR, macrophages/lymphocyte ratio; SII, the systemic immune-inflammation index.

the SP-VATS group, three cases of pleural fluid leakage and one case of pneumothorax in the TP-VATS group (Table 2). The use of analgesics was no difference $(\mathrm{P}=0.607)$ in these two groups (Figure 2).

\section{Postoperative coagulation function and inflammatory markers}

The counts of postoperative PLT $\left[(204.18 \pm 36.91) \times 10^{9} / \mathrm{L}\right.$ vs. $\left.(199.20 \pm 48.47) \times 10^{9} / \mathrm{L}, \mathrm{P}=0.621\right)$ and $\mathrm{D}$-dimer (mean, IQR: $1,425,419-1,992$ vs. 956, 379-1,141 $\mu \mathrm{g} / \mathrm{L} ; \mathrm{P}=0.387$ ) had no difference between SP-VATS group and TP-VATS group. The inflammatory markers included postoperative neutrophil/lymphocyte ratio (NLR), PLT/lymphocyte ratio (PLR), monocyte/lymphocyte ratio (MLR) and SII. The SP-VATS group had less NLR, PLR, MLR and SII compared to TP-VATS group. However, there was no significant difference in these kinds of inflammatory markers.

\section{Discussion}

SP-VATS represents the frontier of VATS. The new generation of thoracic surgeons are enthusiastic about this surgical procedure and the patients are incline to accept it. The present study showed that SP-VATS had better results compared with the TP-VATS, including less operation time and less chest tube. we also found that there was no difference in postoperative complication, intraoperative blood loss, chest tube duration and volume, hospital stays after surgery and postoperative inflammatory response. But it did not verify that SP-VATS was better than TP-VATS in this cohort because of the different surgeons, learning curve (15) and various bias. But we could conclude that SP- 


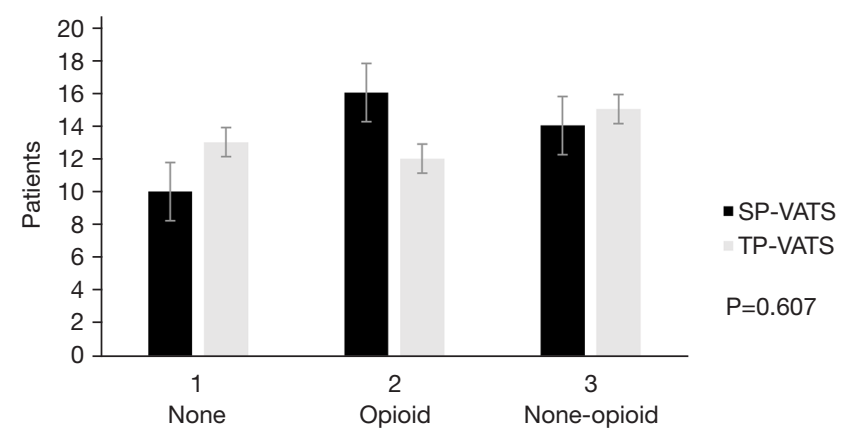

Figure 2 The use of analgesics between SP-VATS and TP-VATS. None-opioid: NASID and tramadol. P value refer to difference of constituent ratio for postoperative analgesia medication of these two groups. SP, single-port; TP, two-port; VATS, video-assisted thoracic surgery.

VATS appeared to be a safe and promising technique and was not inferior to the TP-VATS in our cohort.

The safety and feasibility of SP-VATS for mediastinal surgeries have been well elaborated in a number of studies $(9,16)$. Other studies also reported the similar findings. Li et al. reported that SP-VATS was safe for loco-regional mediastinal lesion resection, correlated with shorter operative time, less intraoperative bleeding and less postoperative pain when compared with multiport techniques (12). Wu et al. found that SP-VATS was associated with a shorter hospital stay and less postoperative pain than conventional VATS in stage I and II thymoma resection (13). In our study, we found that SP-VATS was better than TP-VATS in operating time, hospital stay and drainage duration time, but it did not achieve statistical significance. It may be because the tumor size $(5.73 \pm 2.79$ vs. $6.15 \pm 3.61 \mathrm{~cm} ; \mathrm{P}=0.562)$ was smaller in the SP-VATS and single-port technology made more severe demands on surgeon and assistant. More patients had serious adhesions and more lesion invaded surrounding organs in the TPVATS group. What's more, the studies of Li et al. and $\mathrm{Wu}$ et al. were restricted to thymoma and conventional threeport VATS.

SP-VATS was postulated to reduce postoperative pain as it reduced intercostal nerve injury when compared with TPVATS $(17,18)$. In our center, following the world health organization's "three-step analgesic" principle to manage postoperative pain. Intercostal nerve block and thoracic paravertebral block would be used during operation. Opioid-free and multimodal analgesia are crucial to enhance recovery after surgery and aim to target different pain receptors and pain transmission pathways both peripherally and centrally (19). We measured the use of analgesics for evaluating post-operative pain. But it had no statistical significance. we did not evaluate any pain score to every patient in the perioperative period, thus the objective index for evaluating post-operative pain was not sufficient.

Inflammation is the body's response to endogenous or exogenous injury and playing an important role in the growth of tumors (20). Some studies have demonstrated that systemic inflammatory markers are related to the postoperative outcomes of cancer patients $(21,22)$. Recording the peripheral leukocytes, neutrophils, lymphocytes, macrophages and PLTs to assess the inflammatory response. NLR is a marker for systemic immune response to surgery stimuli (23). PLTs are part of the inflammatory response and benefit to tumour growth, invasion and angiogenesis (24). Lymphocytes are contribution to control tumour growth and improve the prognosis of cancer patients (25). Macrophages can suppress antitumor response and facilitate tumor cell invasion and metastasis by releasing various cytokines and chemokines $(26,27)$. The SII is an objective marker to reflect the local immune response and systemic inflammation (28). Our study showed no difference in NLR, PLR, MLR, SII between the SP and TP group. It revealed that the inflammatory response and exogenous injury caused by these two different surgical approaches were similar. But the prognosis of patients needs to be further researched in our research.

The modern pattern of SP-VATS is short but intense. Since Rocco et al. shared their initial experiences with SPVATS for wedge pulmonary resections in 2004 (17). SPVATS and thoracic anesthetic technology have developed rapidly. In 2013, Gonzalez-Rivas et al. reported the first lobectomy with pulmonary artery reconstruction (29) and the first bronchial sleeve lobectomy (30). In 2014, Gonzalez-Rivas et al. reported the first nonintubated SPVATS lobectomy (31). The combination of modern SPVATS and anesthetic technology has been shinning like a diamond. Nonintubated VATS performs the principle of enhanced recovery after surgery. But the SP-VATS still has some weaknesses. Such as, the operating space between surgeon and assistants was narrow and the VATS instruments fought each other easily. What's more, SPVATS triggers a bigger challenge to young thoracic surgeon. It is worth mentioning that glasses-free 3D VATS help thoracic surgeon to perform better.

Nevertheless, several limitations in our study could not 
be ignored. Firstly, it was a retrospective study. Although propensity matching may reduce the bias, selection bias still existed. Secondly, lack of a complete pain scores. Thirdly, the current study was the single center experience analysis, international multi-center research should be conducted.

\section{Conclusions}

The results of our study show that SP-VATS is safe and feasible for patients with mediastinal tumor, and has potential advantages of less operation time and less chest tube when compared with TP-VATS.

\section{Acknowledgments}

Funding: This work was supported by the following funding: the grant 2016YFC0905400 from the National Key R\&D Program of China; China National Science Foundation (Grant No. 81871893 \& No. 81501996); Key Project of Guangzhou Scientific Research Project (Grant No. 201804020030); High-level university construction project of Guangzhou medical university (Grant No. 20182737, 201721007, 201715907, 2017160107); National key R \& D Program (Grant No. 2017YFC0907903 \& 2017YFC0112704).

\section{Footnote}

Conflicts of Interest: The authors have no conflicts of interest to declare.

Ethical Statement: The authors are accountable for all aspects of the work in ensuring that questions related to the accuracy or integrity of any part of the work are appropriately investigated and resolved. The National Key R\&D Program of China evaluated the protocol. The data involved in this study was collected retrospectively, which was not required the statement of ethics approval.

\section{References}

1. Odaka M, Shibasaki T, Kato D, et al. Comparison of oncological results for early- and advanced-stage thymomas: thoracoscopic thymectomy versus open thymectomy. Surg Endosc 2017;31:734-42.

2. Hwang SK, Park SI, Kim YH, et al. Clinical results of surgical resection of mediastinal teratoma: efficacy of video-assisted thoracic surgery. Surg Endosc
2016;30:4065-8.

3. Flores RM, Park BJ, Dycoco J, et al. Lobectomy by videoassisted thoracic surgery (VATS) versus thoracotomy for lung cancer. J Thorac Cardiovasc Surg 2009;138:11-8.

4. Bendixen M, Jorgensen OD, Kronborg C, et al. Postoperative pain and quality of life after lobectomy via video-assisted thoracoscopic surgery or anterolateral thoracotomy for early stage lung cancer: a randomised controlled trial. Lancet Oncol 2016;17:836-44.

5. Roviaro G, Rebuffat C, Varoli F, et al. Videoendoscopic pulmonary lobectomy for cancer. Surg Laparosc Endosc 1992;2:244-7.

6. Landreneau RJ, Mack MJ, Hazelrigg SR, et al. Videoassisted thoracic surgery: basic technical concepts and intercostal approach strategies. Ann Thorac Surg 1992;54:800-7.

7. Yim AP, Ho JK, Chung SS, et al. One hundred and sixtythree consecutive video thoracoscopic procedures: the Hong Kong experience. Aust N Z J Surg 1994;64:671-5.

8. Yamamoto H, Okada M, Takada M, et al. Video-assisted thoracic surgery through a single skin incision. Arch Surg 1998;133:145-7.

9. Mineo TC, Ambrogi V. A glance at the history of uniportal video-assisted thoracic surgery. J Vis Surg 2017;3:157.

10. Shen Y, Wang H, Feng $M$, et al. Single- versus multipleport thoracoscopic lobectomy for lung cancer: a propensity-matched studydagger. Eur J Cardiothorac Surg 2016;49 Suppl 1:i48-53.

11. Masmoudi H, Etienne H, Sylvestre R, et al. Three Hundred Fifty-One Patients With Pneumothorax Undergoing Uniportal (Single Port) Video-Assisted Thoracic Surgery. Ann Thorac Surg 2017;104:254-60.

12. Li Q, Sihoe A, Wang H, et al. Short-term outcomes of single- versus multi-port video-assisted thoracic surgery in mediastinal diseases. Eur J Cardiothorac Surg 2018;53:216-20.

13. Wu CF, Gonzalez-Rivas D, Wen CT, et al. Comparative Short-Term Clinical Outcomes of Mediastinum Tumor Excision Performed by Conventional VATS and SinglePort VATS: Is It Worthwhile? Medicine (Baltimore) 2015;94:e1975.

14. Trotti A, Colevas AD, Setser A, et al. CTCAE v3.0: development of a comprehensive grading system for the adverse effects of cancer treatment. Semin Radiat Oncol 2003;13:176-81.

15. Martin-Ucar AE, Aragon J, Bolufer Nadal S, et al. The influence of prior multiport experience on the learning 
curve for single-port thoracoscopic lobectomy: a multicentre comparative studydagger. Eur J Cardiothorac Surg 2017;51:1183-7.

16. Xie D, Wang H, Fei K, et al. Single-port video-assisted thoracic surgery in 1063 cases: a single-institution experiencedagger. Eur J Cardiothorac Surg 2016;49 Suppl 1:i31-6.

17. Rocco G, Martin-Ucar A, Passera E. Uniportal VATS wedge pulmonary resections. Ann Thorac Surg 2004;77:726-8.

18. Jutley RS, Khalil MW, Rocco G. Uniportal vs standard three-port VATS technique for spontaneous pneumothorax: comparison of post-operative pain and residual paraesthesia. Eur J Cardiothorac Surg 2005;28:43-6.

19. Beverly A, Kaye AD, Ljungqvist O, et al. Essential Elements of Multimodal Analgesia in Enhanced Recovery After Surgery (ERAS) Guidelines. Anesthesiol Clin 2017;35:e115-43.

20. Diakos CI, Charles KA, McMillan DC, et al. Cancerrelated inflammation and treatment effectiveness. Lancet Oncol 2014;15:e493-503.

21. Ishizuka $M$, Nagata $H$, Takagi $K$, et al. Inflammationbased prognostic score is a novel predictor of postoperative outcome in patients with colorectal cancer. Ann Surg 2007;246:1047-51

22. Wei XL, Wang FH, Zhang DS, et al. A novel inflammation-based prognostic score in esophageal squamous cell carcinoma: the C-reactive protein/albumin ratio. BMC Cancer 2015;15:350.

23. Turner N, Wong HL, Templeton A, et al. Analysis of

Cite this article as: $\mathrm{Wu} \mathrm{S}$, Liang $\mathrm{H}$, Liang $\mathrm{W}$, Zhang $\mathrm{Y}, \mathrm{Ma}$ Y, Liu H, Yang H, Liu J, He J. Single- versus two-port videoassisted thoracic surgery in mediastinal tumor: a propensitymatched study. J Thorac Dis 2019;11(11):4428-4435. doi: $10.21037 /$ jtd.2019.11.05 local chronic inflammatory cell infiltrate combined with systemic inflammation improves prognostication in stage II colon cancer independent of standard clinicopathologic criteria. Int J Cancer 2016;138:671-8.

24. Kusumanto YH, Dam WA, Hospers GA, et al. Platelets and granulocytes, in particular the neutrophils, form important compartments for circulating vascular endothelial growth factor. Angiogenesis 2003;6:283-7.

25. Ferrone C, Dranoff G. Dual roles for immunity in gastrointestinal cancers. J Clin Oncol 2010;28:4045-51.

26. Elgert KD, Alleva DG, Mullins DW. Tumor-induced immune dysfunction: the macrophage connection. J Leukoc Biol 1998;64:275-90.

27. Pollard JW. Tumour-educated macrophages promote tumour progression and metastasis. Nat Rev Cancer 2004;4:71-8.

28. Huang H, Liu Q, Zhu L, et al. Prognostic Value of Preoperative Systemic Immune-Inflammation Index in Patients with Cervical Cancer. Sci Rep 2019;9:3284.

29. Gonzalez-Rivas D, Delgado M, Fieira E, et al. Single-port video-assisted thoracoscopic lobectomy with pulmonary artery reconstruction. Interact Cardiovasc Thorac Surg 2013;17:889-91.

30. Gonzalez-Rivas D, Fernandez R, Fieira E, et al. Uniportal video-assisted thoracoscopic bronchial sleeve lobectomy: first report. J Thorac Cardiovasc Surg 2013;145:1676-7.

31. Gonzalez-Rivas D, Fernandez R, de la Torre M, et al. Single-port thoracoscopic lobectomy in a nonintubated patient: the least invasive procedure for major lung resection? Interact Cardiovasc Thorac Surg 2014;19:552-5. 